\title{
Application of Spectrum-Subtracted Sub-band Singular Spectrum Entro- py in Sediment Thickness Measurement
}

\author{
Di Fan ${ }^{1,2, *}$ Lu Wei ${ }^{1}$ and Maoyong Cao ${ }^{1,2}$ \\ ${ }^{1}$ Shandong University of Science and Technology, Qingdao, 266590, China \\ ${ }^{2}$ Shandong Provincial Key Laboratory of Robotics and Intelligent Technology, Qingdao, China
}

\begin{abstract}
Obtaining the wave head location and acoustic time difference with high accuracy in measuring sediment thickness in borehole by ultrasonic is a key and hard problem. The methods available now can't meet the precision and noise immunity requirement. This paper utilizes the good performance of Spectrum-Subtracted sub-Band Singular Spectrum Entropy (SSB-SSE) to process the echoes reflected from the sediment and get their wave head positions. Based on these obtained data, acoustic time difference can be calculated, then the sediment thickness can be achieved. The results of three typical experiments show that SSB-SSE is very suitable in locating the wave heads of thicker sediment layer's echoes, and it also performs well even when the echo is covered by noise.
\end{abstract}

Keywords: Spectrum-Subtracted sub-Band Singular Spectrum Entropy (SSB-SSE), Wave head location, Sediment thickness, Ultrasonic.

\section{INTRODUCTION}

Using the time difference between the two echoes of the sediment layer is a basic method to measure the medium thickness or distance. It also can be adopted to measure the sediment thickness in borehole. As for obtaining the ultrasonic time difference of the sediment layer, there are some basically methods such as time delay estimation based on Wiener filtering [1] and wave head locating based on local Signal Noise Ratio (SNR) [2]. The former method can get good result when the sediment layer is thin and the two echoes' amplitudes are close. But when the sediment layer is thick, it will be invalid for the echo reflected from the sediment's lower interface is too weak. The later method not only can't get high precision wave head location but also is easy to be influenced by noise.

Sediment thickness is proportional to the ultrasonic travel time in the sediment. The priority work in obtaining ultrasonic travel time (equal to sound time difference) is to get the time when the echoes' wave head arrive (that is the wave head location in time axis). If wave heads of the echoes reflected from the sediment layer's two interfaces arrive at $t_{1}$ and $t_{2}$ respectively, then the ultrasonic travel time in the sediment, or sound time difference, is $! t=t_{2} \quad t_{1}$. Assuming the ultrasonic speed in the liquid in sediment is $v$, then the sediment thickness is: $d=v ! \quad t / 2$

Unfortunately, wave head is usually very weak and always easy to be confused by noise [3], in addition, its amplitude will damp very quickly with the acoustic source central frequency increasing [4]. So, as for the sediment thickness measurement, that the ultrasonic probe must work at intermediate or high frequency will worsen locating the wave head.

Presently, existing methods for wave head detection are mainly thresholding method [5], windowed correlation-peak method [6], long \& short time-windowed energy ratio method [3], wavelet transform modulus maxima method [7], and Hilbert-Huang transform method [8]. Only when the echo's Signal Noise Ratio (SNR) is high, especially the local SNR near the wave head, these methods can work effectively. Otherwise, their performances will much degrade. Moreover, the locating accuracy of these methods are not high enough and meanwhile its sensitivity to the echo's amplitude also much reduces its anti-jamming capability. Combining the technologies of end-point detecting method in voice processing and singularity detection method in mechanical fault diagnosis, reference $[9,10]$ have developed a new wave head locating method based on Spectrum-Subtracted sub-Band Singular Spectrum Entropy (SSB-SSE). The simulation results of this method have also demonstrated its features in accuracy, robustness and anti-jamming capability.

This paper applied SSB-SSE in sediment thickness measurement by detecting and locating the ultrasonic echoes' wave heads. Using the ultrasonic signals of sediment with different thickness obtained in lab environment, three experiments have been operated to locate the echoes' wave 
heads and measure the sediment thickness. The experimental results demonstrate that SSB-SSE with good advantages in terms of robustness to the signal's amplitude can locate the weak wave head even when it's covered by noise under the thick sediment condition.

\section{SPECTRUM-SUBTRACTED SUB-BAND SIN- GULAR SPECTRUM ENTROPY}

As for a $N$-point signal sequence $x(k)$, according to the

Phase Space Reconstruction Theory [11], when a positive integer $n$ less than $N$ is selected, a Hankle matrix $A$ can be constructed from $x(k)$ as:

$$
A=\left[\begin{array}{cccc}
x(1) & x(2) & \cdots & x(N-n+1) \\
x(2) & x(3) & \cdots & x(N-n+2) \\
\vdots & \vdots & \vdots & \vdots \\
x(n) & x(n+1) & \cdots & x(N)
\end{array}\right]
$$

$A$ is an $m ! n$ matrix, where $m=N-n+1$. Singular value decomposition of $A$ is:

$$
A=U P V^{\mathrm{H}} \text {, }
$$

where, superscript " $H$ " is conjugate transpose operator to matrix; $U$ and $V$ are $m \times m$ and $n \times n$ unitary matrixes respectively. When $r=\min (m, n) \quad, \quad \Sigma=\operatorname{diag}\left(\sigma_{1}\right.$, $\left.\sigma_{2}, \sigma_{3}, \cdots \cdot \sigma_{r}\right)$, and $O_{k \times l}$ is a $k \times l$ zero matrix. If $m=n$, then $P=\Sigma$; if $m>n$, then $P=\left[\begin{array}{c}\Sigma_{n \times n} \\ O_{(m-n) \times n}\end{array}\right]$, and if $m<n$, then $P=\left[\begin{array}{lll}\Sigma_{m \times m} & O & \\ & & \\ m \times(n-m)\end{array}\right]$.

$\sigma_{i}(i=1,2, \cdots \cdots, r)$, non-negative value, is named the singular value of matrix $A$, also called Singular Spectrum (SS). They are generally arranged in descending order as:

$\sigma_{1}>\sigma_{2}>\sigma_{3}>\cdots \cdots, \sigma_{r-1}>\sigma_{r}$

As for any common signal, its Hankle matrix is always low rank, that is, only very few singular values are large, and the others are almost zero. As for a random noise, its Hankle matrix is usually full rank, theoretically the singular values are its variance, that is

$\Sigma_{n}=\delta E$

here $E$ is an unit matrix.

Spectrum-Subtracted sub-Band Singular Spectrum Entropy takes advantages of both Spectrum-Subtracted and sub-Band with Singular Spectrum Entropy (SSE). That is, firstly apply spectrum-subtracted processing to remove noise, and then divide the singular spectrum into several subbands, after that, calculate sub-band Spectrum Entropy.
Although the Hankle matrix of a noisy signal is full rank, it also has a few large singular values. If the singular values of noise can be estimated and subtracted from the entire singular values, the signal's singular values with high SNR can be gotten. Assuming noise's singular values are $\sigma_{n i}(i=1,2, \cdots \cdots r)$ and the noisy signal's are $\sigma_{i}^{\prime}(i=1,2, \cdots \cdots r)$.

Spectrum-Subtracted sub-Band Singular Spectrum Entropy proposed in this paper employs both noise's singular values subtraction and sub-band to SSE. For processing of the noise's singular values subtraction, the SS in the following equations of SSB-SSE is $\sigma_{i}=\sigma_{i}^{\prime}-\sigma_{n i}(i=1,2, \cdots \cdots r)$.

A sub-band of singular spectrum is defined as a subset which includes certain singular values. Suppose dividing all the singular values into $M$ sub-bands and the number of singular values in $m$-th sub-band is $N_{m}, m=1,2, \cdots, M$, then the total energy of each sub-band is

$$
E_{m}=-\sum_{i=s_{m}+1}^{s_{m}+N_{m}} \sigma_{i}
$$

where $s_{m}=\sum_{i=1}^{m-1} N_{i}$. Then the PDF of sub-Band Singular Value Spectrum is defined as:

$p_{s}(k)=E_{k} / \sum_{j=1}^{M} E_{j}, k=1,2, \cdots, M$

Sub-Band Singular Value Spectrum Entropy(B-SSE) is

$H_{s}=-\sum_{i=1}^{M} p_{s}(i) \log \left[p_{s}(i)\right]$

In practical application, in view of some PDF of the singular values may be zero, the formula above is transformed as:

$$
H_{s}=-\sum_{i=1}^{M} p_{s}(i) \log \left[1+p_{s}(i)\right]
$$

According to the physical characteristics of ultrasonic transducer, ultrasonic signal is an oscillation process and its singular values are always paired. Therefore, the sub-band of its singular value spectrum should be divided by pairs. So, after sorting the singular value by descending order, divide every pair of singular values into one sub-band. This division of sub-band is reasonable for it well matching with the features of the ultrasonic signal's singular spectrums.

\section{APPLICATION EXPERIMENTS}

In our lab, a circular bucket with diameter of about $75 \mathrm{~cm}$ is used as simulated borehole. Sand is placed on the bottom of the bucket filled with water to simulate sediment. Ultrasonic transducer is a transmit-receive one with bandwidth of $80-120 \mathrm{kHz}$. In experiments, transducer is put into the water and sends ultrasound with certain frequency vertically to the 


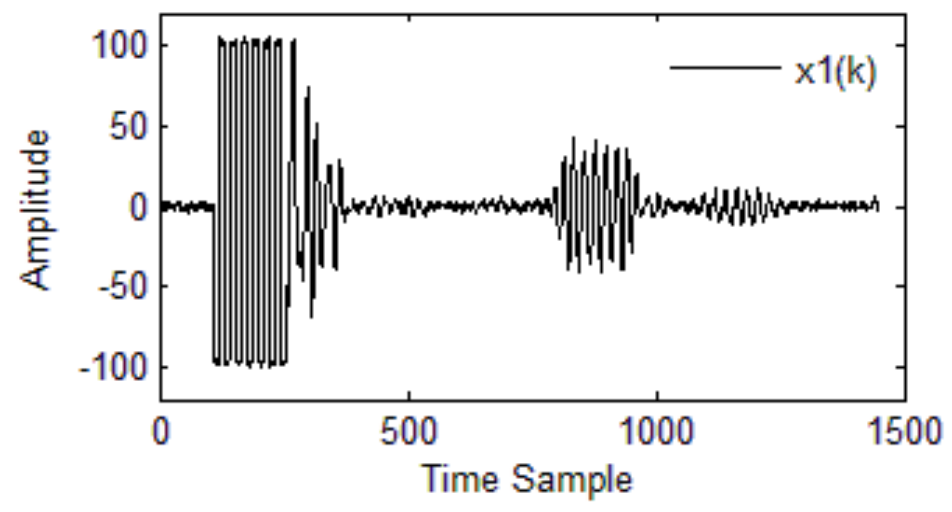

(a) Ultrasonic signal $x l(k)$.

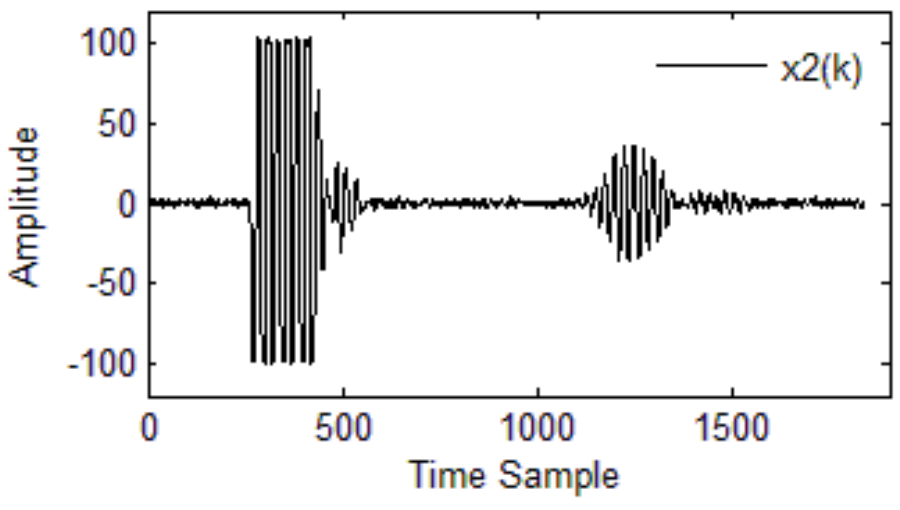

(b) Ultrasonic signal $x 2(k)$.

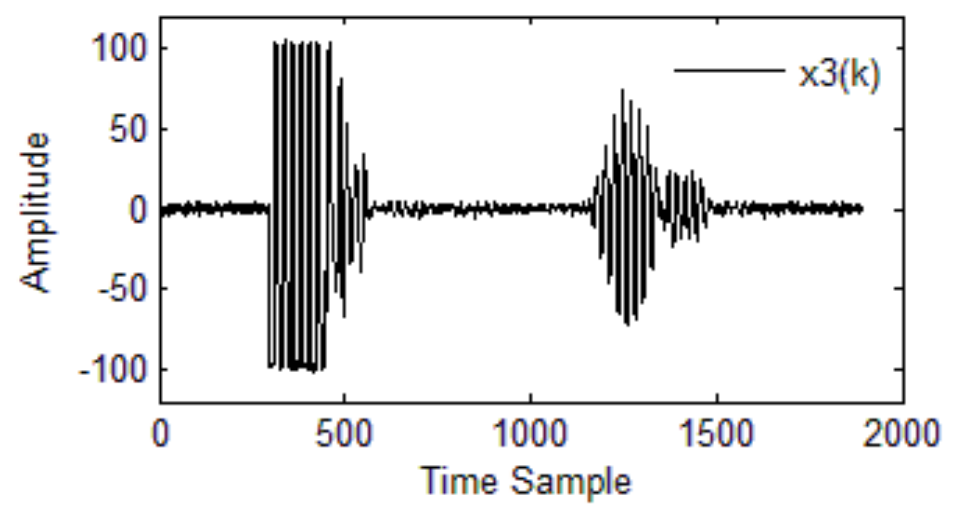

(c) Ultrasonic signal $x 3(k)$.

Fig. (1). The ultrasonic echoes reflected from sediment of different thickness.

sand. The echoes carrying the information of sediment thickness are received by the transducer, as shown in Fig. (1). In Fig. (1a), named $x l(k)$, is an echoes signal obtained under the condition with the sediment thickness of about $11.51 \mathrm{~cm}$ and the ultrasonic central frequency of $90 \mathrm{KHz}$. In Fig. (1b), named $x 2(k)$, is obtained under the condition with the sediment thickness of about $10.10 \mathrm{~cm}$ and the ultrasonic central frequency of $80 \mathrm{KHz}$. In Fig. (1c), named $x 3(k)$, is obtained when sediment thickness of about $7.82 \mathrm{~cm}$ and the ultrasonic central frequency of $90 \mathrm{KHz}$. These three signals are all sampled with the sampling frequency of $2 \mathrm{MHz}$.
The method how to estimate sediment thickness is as follows: calculate the SSB-SSE of the ultrasonic echoes reflected from the sediment, and locate the sub-wave's wave head with the threshold of SSB-SSE and then calculate the time difference of the echoes and sediment thickness. The adopted threshold $T h$ is:

$T h=a \times\left[\max \left(H_{s}(k)\right)-\min \left(H_{s}(k)\right)\right]+\min \left(H_{s}(k)\right)$

where, $H_{s}(k)$ is the signal's SSB-SSE sequence; $\max ()$ and $\min ()$ are the functions of getting the maximum and mini- 


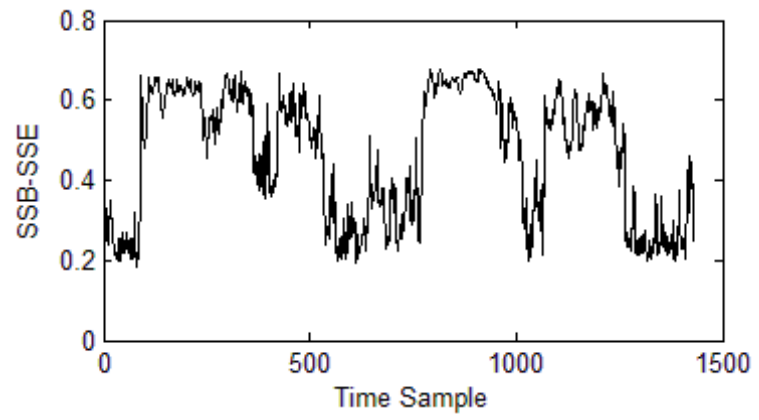

(a) The SSB-SSE.

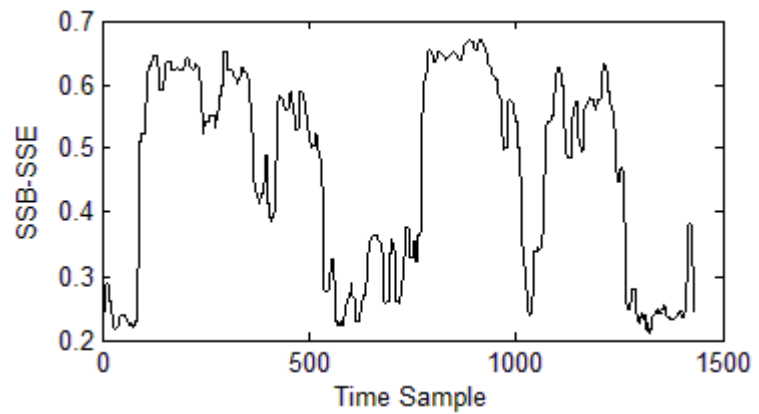

(b) The SSB-SSE after being median filtered.

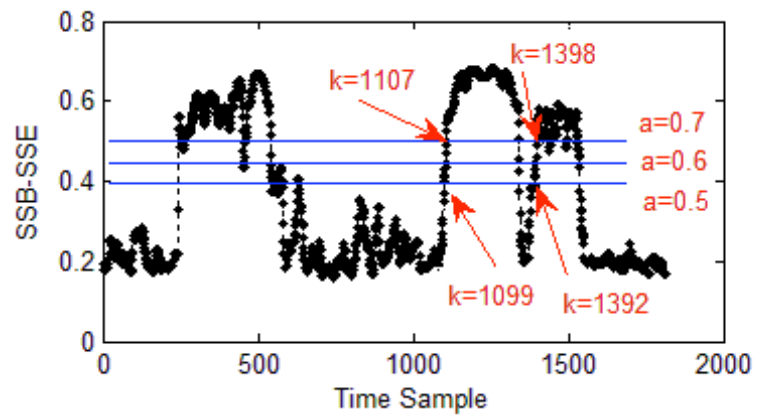

(c) The wave head locations.

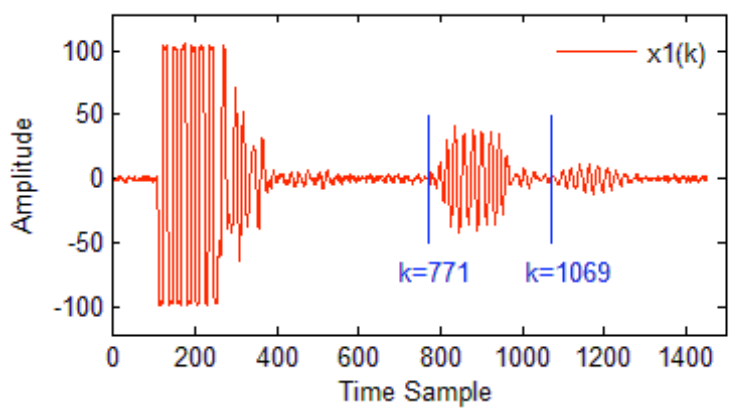

(d) The wave head locations in the signal.

Fig. (2). The SSB-SSE and its wave head locations in $x l(k)$.

mum respectively; $a$ is a scalar factor, through which to adjust the threshold height, larger $a$ makes a larger threshold.

Experiment 1: This experiment is for the echoes signal $x 1(k)$ in Fig. (1a). Because the central frequency of the signal is $90 \mathrm{KHz}$, and the sampling frequency is $2 \mathrm{MHz}$, the discrete signal's cycle is about 22 points, and so the analysis window's length $N w$ should be integer times of 22. Fig. (2a) and Fig. (2d) show the signal's SSB-SSE $(N w=22)$ and the result after it's filtered by 17 -points median filter, the detail of wave head locations and the wave head locations in the original signal.

From the data in Fig. (2c), when threshold coefficient $a$ is in the range of 0.5-0.7, the wave head location of the two sub-waves of the sediment's interfaces is about 771-773 and 


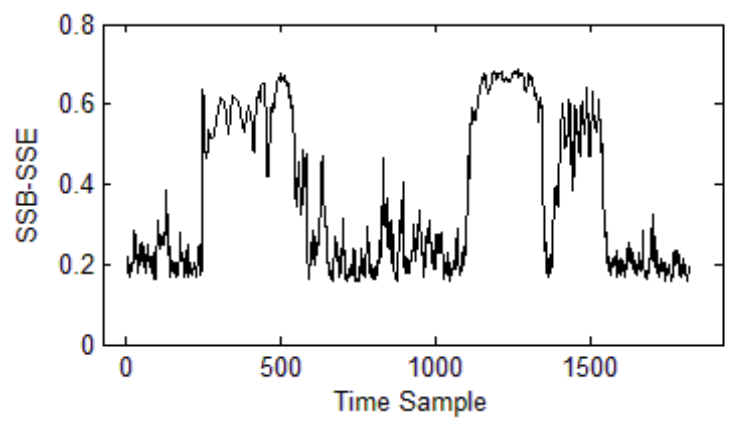

(a) The SSB-SSE.

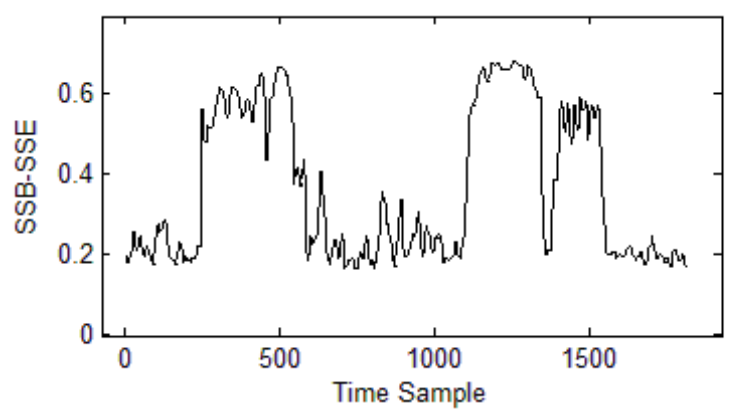

(b) The SSB-SSE after being median filtered.

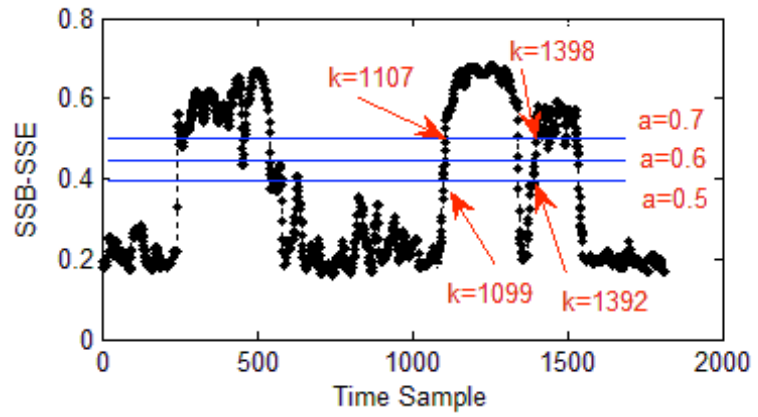

(c) Wave head locations.

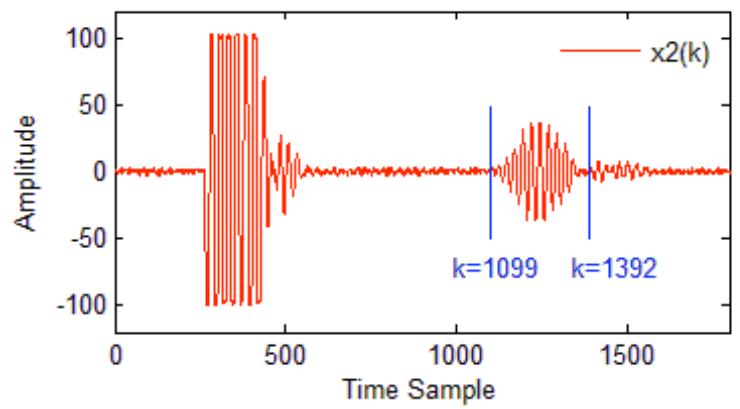

(d) The wave head locations in the signal.

Fig. (3). The SSB-SSE and its wave head locations in $x 2(k)$.

1069-1071 respectively, where the location of 771 and 1069 in original signal are shown in Fig. (2d) by blue vertical lines. So, even the threshold changes in a rather large range, the wave head position located by SSB-SSE changes very small. In this experiment, when threshold changes from 0.5 to 0.7 , the locating results only change 3 points (equal to thickness of $0.1 \mathrm{~cm}$ ). This illustrates the robustness of the SSB-SSE in locating the wave head. From Fig. (2d), when the wave head is very weak even covered by noise, SSB-SSE can also locate the wave head correctly, these locating results are hard to get by other methods. 


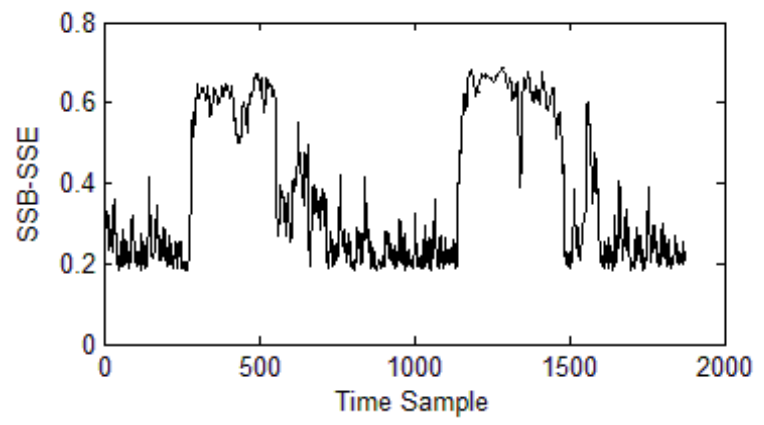

(a) The SSB-SSE.

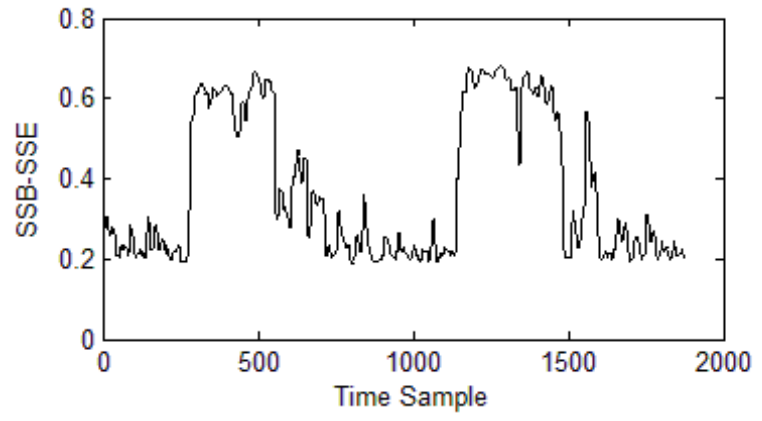

(b) The SS-B-SSE after being median filtered.

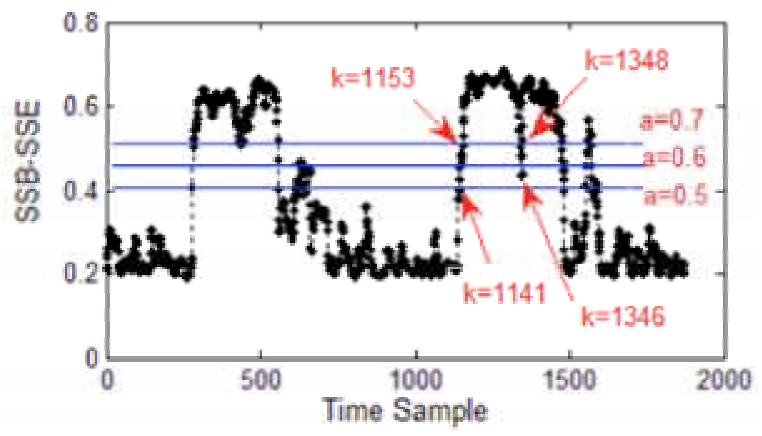

(c) Wave head locations.

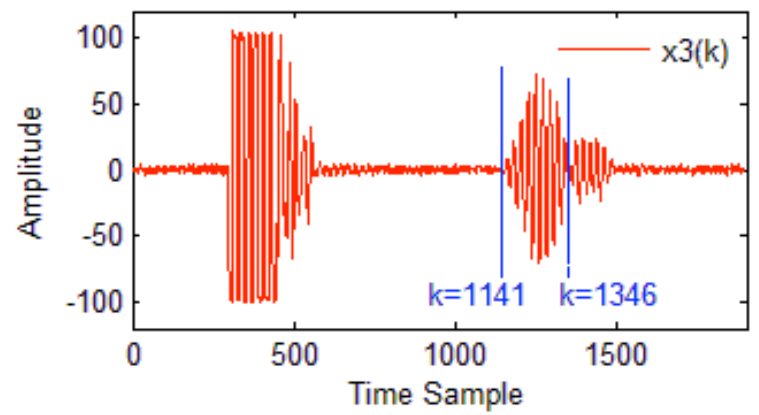

(d) The wave head locations in the signal.

Fig. (4). The SSB-SSE and its wave head locations in $x 3(k)$.

When using the threshold of $a=0.6$ to locate the wave head and estimate the sediment thickness, the time difference between the two sub-waves is $\Delta t=(1070-772) \times T=298 * 0.5 \mu s=149.0 \mu s$, where, $T$ is sampling interval, since sampling frequency is $2 \mathrm{MHz}, T=0.5 \mu \mathrm{s}$. The ultrasonic speed in water is about $1500 \mathrm{~m} / \mathrm{s}$, if we use this speed, then the sediment thickness is $d=0.5 \times \Delta t \times v=11.175 \mathrm{~cm}$. 
Table 1. Summary of the experimental conditions and results. (Note: Expt 1 stands for the experiment 1, indicating where the data in this column come from. So do Expt 2 and Expt 3).

\begin{tabular}{|c|c|c|c|}
\hline Items & Expt 1 & Expt 2 & Expt 3 \\
\hline $\begin{array}{c}\text { Thickness measured by } \\
\text { ruler }(\mathrm{cm})\end{array}$ & 11.5 & 11.1 & 7.8 \\
\hline $\begin{array}{l}\text { Ultrasonic Central fre- } \\
\text { quency }(\mathrm{kHz})\end{array}$ & 90 & 80 & 90 \\
\hline Window length (point) & 22 & 24 & 22 \\
\hline $\begin{array}{c}\text { Wave head in Sub-wave } \\
1 \text { (point) }\end{array}$ & $771-773$ & $1099-1107$ & $1141-1153$ \\
\hline $\begin{array}{c}\text { Wave head in Sub-wave } \\
2 \text { (point) }\end{array}$ & $1069-1071$ & $1392-1398$ & $1336-1348$ \\
\hline $\begin{array}{l}\text { Wave head in two sub- } \\
\text { waves (point) }\end{array}$ & 772,1070 & 1105,1394 & 1143,1337 \\
\hline $\begin{array}{l}\text { Acoustic time difference } \\
\qquad(\mu s, \mathrm{a}=0.6)\end{array}$ & 149 & 144.5 & 97 \\
\hline $\begin{array}{l}\text { Thickness got by SSB- } \\
\text { SSE }(\mathrm{cm})\end{array}$ & 11.12 & 10.84 & 7.28 \\
\hline
\end{tabular}

Experiment 2: This experiment is for the echoes signal $x 2(k)$ in Fig. (1b). Here the central frequency of the signal is $80 \mathrm{KHz}$, with the sampling frequency of $2 \mathrm{MHz}$, the discrete signal's cycle is about 24 points. So the analysis window's length $N w$ should be integer times of 24. Fig. (3a) and Fig. (3d) show the signal's SSB-SSE $(N w=24)$ and the result after it's filtered by 17-points median filter respectively, the detail of wave head locations and the wave head locations in the original signal.

From the data in Fig. (3c), when threshold coefficient $a$ is in the range of $0.5-0.7$, the wave head locations of the two sub-waves of the sediment's interfaces are about 1099-1107 and 1392-1398 respectively, where the locations of 1099 and 1392 in original signal are shown in Fig. (3d) by blue vertical lines. In this experiment, when threshold changes from 0.5 to 0.7 , the locating results change maximum 8 points (equal to thickness of $0.3 \mathrm{~cm}$ ). This varying range is larger than that in experiment 1 , the main reason is because the sediment thickness is smaller than that in experiment 1 . The thinner sediment makes the two echoes of the sediment's interfaces close enough to be covered by the analysis window at the same time when it slides on the analysis window doesn't cover either echoes. From Fig. (2d), even with difficulties, SSB-SSE can locate the wave head with rather high accuracy.

When using the threshold of $a=0.6$, the two sub-waves' wave head locations are 1105 and 1394, with the same sampling interval $T$ and ultrasound speed as experiment 1 , then the time difference sediment thickness is as follows:

$\Delta t=(1394-1105) \times T=289 * 0.5 \mu s=144.5 \mu s$, $d=0.5 \times \Delta t \times v=0.5 \times 144.5 \mu s \times 1500 \mathrm{~m} / \mathrm{s}=10.8375 \mathrm{~cm}$

Experiment 3: This experiment is for the echoes signal $x 3(k)$ in Fig. (1c). Since the ultrasonic central frequency and the sampling frequency are all the same as that in experiment 1 , the discrete signal's cycle is also about 22 points, and the analysis window's length $N w$ should be integer times of 22 . Fig. (4a) and Fig. (4d) show the signal's SSB-SSE $(N w=22)$ and the result after it's filtered by 17-points median filter respectively, the detail of wave head locations and the wave head locations in the original signal.

From the data in Fig. (4c), when threshold coefficient $a$ is in the range of $0.5-0.7$, the wave head locations of the two sub-waves of the sediment's interfaces are about 1141-1153 and 1336-1348 respectively, where the locations of 1141 and 1336 in original signal are shown in Fig. (4d) by blue vertical lines. In this experiment, when threshold changes from 0.5 to 0.7 , the locating results change maximum 12 points (equal to thickness of $0.45 \mathrm{~cm}$ ). This varying range is larger than those in the two previous experiments. The main reason is also because the sediment thickness is the smallest in the three experiments. The two echoes of the sediment's interfaces are much closer now and the analysis window also will covers both echoes at the same time during its sliding on the echoes. This covering brings error to the locating. In experiment 1, the two echoes are separated by a big space, the analysis window doesn't cover either echoes. From Fig. (2d), even with difficulties, SSB-SSE can locate the wave head with rather high accuracy. Even though, from Fig. (2d) we can see SSB-SSE also can locate the weak wave head positions with high precision.

When using the threshold of $a=0.6$, the two sub-waves' wave head locations are 1105 and 1394, with the same sampling interval $T$ and ultrasound speed as experiment 1 , then the time difference sediment thickness is as follows:

$$
\begin{aligned}
& \Delta t=(1337-1143) \times T=194 * 0.5 \mu \mathrm{s}=97 \mu \mathrm{s}, \\
& d=0.5 \times \Delta t \times v=0.5 \times 97 \mu \mathrm{s} \times 1500 \mathrm{~m} / \mathrm{s}=7.275 \mathrm{~cm} .
\end{aligned}
$$

Now we list all the conditions and results of the above three experiments in the Table $\mathbf{1}$.

\section{CONCLUSION}

This paper applies the SSB-SSE in sediment thickness measurement. By locating the ultrasonic wave heads, we get the time difference of the two echoes and then estimate the sediment thickness.

From the experiments, when the sediment is thicker and the time difference of the two echoes is larger, the locating results of wave heads are more accurate. With the sediment thickness decreasing, the time difference of the two echoes reduces. When the space between the two echoes is less than the analysis window's length, the former echo must affect the locating of the later echo head wave. The smaller the space is the bigger the affection will be. Nevertheless, in general, SSB-SSE can detect and locate the weak wave head 
of the echoes, and based on the wave head location, the sediment thickness can be estimate with high precision.

It is worth mentioning that using multi-windows and multi-window correction can further improve the wave head locating accuracy and solve the measurement of the thin sediment.

\section{CONFLICT OF INTEREST}

The authors confirm that this article content has no conflict of interest.

\section{ACKNOWLEDGEMENTS}

This paper belongs to the project of the "Shandong Province Young Scientist Foundation", No. BS2012DX034; "China Postdoctoral Science Foundation", No. 2012 M521361; "Shandong Province Natural Science Foundation", No. ZR2012EEM021; "Project of Shandong Province Higher Educational Science and Technology Program", No. J13LN17; "Innovation Fund for Graduate Students of SDUST", No.YC140212; "Project of South Africa/China Research Collaboration in Science and Technology", No. 2012DFG71060.

\section{REFERENCES}

[1] D. Fan, M. Y. Cao, and N. L. Sun, "Time delay estimation based on wiener filer in ultrasonic detection of sediments in drilling hole", In: Proceedings of IEEE Second International
Workshop on Computer Science and Engineering (WCSE09), Oct. 2009, pp. 582-585.

[2] D. Fan, M. Y. Cao, N. L. Sun, C. Z. Lv, and Q. Z. Zhao, "Locating the Ultrasonic Echo Head Wave of Bottom Sediment in Drilling Hole Based on Local SNR", In: Proceedings of The $2^{\text {nd }}$ International Conference on Image and Signal Processing (CISP2009), 2009, pp. 4780-4783.

[3] J. W. Zhang, Y. B. Shi, Z. G. Wang, and X. E. Liu, "Research of signal process and detecting head wave of acoustic array", China Measurement Technology, vol. 32, no.4, pp. 4-5,100, July 2006.

[4] Y. Q. Zhou, X. M. Wang, and D. H. Chen, "Study of numerical simolation on amplitude attenuation of first arrivals in cased-hole acoustic logging", Well Logging Technology, vol. 31, no.4, pp. 321-326, Aug 2007.

[5] W. W. Huang, G. P. Shao, and J. X. Li, "Detection and correlation correct of first arrival in digital acoustic array", Well Logging Technology, vol. 29, no.2, pp. 112-114, June 2005.

[6] H. Chen, and Z. M. Zhao, "Extraction of head wave energy for formation wave in cement bond evaluation", Petroleum Instruments, vol.19, no.6, pp. 46-47, Dec 2005.

[7] Y. Song, H. Y. Zhen, K. G. Chen, X. H. Liu, and L. G. Peng, and Q.Y. Zheng, "Detection of shear head wave of dipole acoustic logging based on wavelet transformation", Journal of Oil and Gas Technology, vol. 30, no.5, pp. 73-76, Oct 2008.

[8] Q. G. Li, and S. W. Tan, "A comparative study on the check of signal singularity based on hilber-huang transform", Journal of Xihua University Natural Science, vol. 27, no. 4, pp. 3-6, Oct 2008.

[9] D. Fan, "Study on signal processing technology and their applications in ultrasonic detection of sediments thickness", $\mathrm{PhD}$ thesis, Shandong University of Science and Technology, Shandong, China, June 2010.

[10] M. Y. Cao, D. Fan, H. B. Liang, and N. L. Sun, "A method and equipment of detecting and locating the ultrasonic wave head", Chinese Patent ZL201010135383.5, July, 2012.

[11] W. William, and A. M. Wu, "Nonlinear singular spectrum analysis", Neural Networks, vol. 3, pp. 2819-2824, May 2002.

(C) Fan et al.; Licensee Bentham Open.

This is an open access article licensed under the terms of the Creative Commons Attribution Non-Commercial License (http://creativecommons.org/licenses/by$\mathrm{nc} / 3.0 /$ ) which permits unrestricted, non-commercial use, distribution and reproduction in any medium, provided the work is properly cited. 